\title{
BMJ Open Efficacy of anti-TNF dosing interval lengthening in adolescents and young adults with inflammatory bowel disease in sustained remission (FREE-study): protocol for a partially randomised patient preference trial
}

\author{
Marleen Bouhuys (D) , Willem S Lexmond, ${ }^{1}$ Gerard Dijkstra, ${ }^{2}$ Triana Lobatón, ${ }^{3}$ \\ Edouard Louis, ${ }^{4}$ Stephanie van Biervliet, ${ }^{5}$ Henk Groen (1) , ${ }^{6}$ Jordi Guardiola, ${ }^{7}$ \\ Patrick van Rheenen (D) ${ }^{1}$
}

To cite: Bouhuys $M$, Lexmond WS, Dijkstra G, et al. Efficacy of anti-TNF dosing interval lengthening in adolescents and young adults with inflammatory bowel disease in sustained remission (FREE-study): protocol for a partially randomised patient preference trial. BMJ Open 2021;11:e054154. doi:10.1136/ bmjopen-2021-054154

- Prepublication history for this paper is available online To view these files, please visit the journal online (http://dx.doi org/10.1136/bmjopen-2021054154).

Received 03 June 2021 Accepted 15 0ctober 2021

Check for updates

(C) Author(s) (or their employer(s)) 2021. Re-use permitted under CC BY-NC. No commercial re-use. See rights and permissions. Published by BMJ.

For numbered affiliations see end of article.

Correspondence to Dr Patrick van Rheenen; p.f.van.rheenen@umcg.nl

\section{ABSTRACT}

Introduction Anti-tumour necrosis factor (TNF) therapy has greatly improved treatment outcomes in patients with inflammatory bowel disease (IBD), but long-term use is associated with cutaneous reactions, susceptibility to infections and frequent injections or hospital visits. Several non-controlled studies have demonstrated that dose reduction is feasible for a subset of patients, provided that early detection of a disease flare is possible. Here, we aim to compare the effectiveness of interval lengthening with standard dosing in maintaining remission in young patients with IBD.

Methods and analysis In this international, prospective, non-inferiority, partially randomised patient preference trial, we aim to recruit 148 patients aged 12-25 years with luminal Crohn's disease or ulcerative colitis in sustained remission (ie, three consecutive in-range faecal calprotectin $(\mathrm{FC})$ results or recently confirmed endoscopic remission). In the interventional arm, the dosing interval will be lengthened from 8 to 12 weeks for infliximab users and from 2 to 3 weeks for adalimumab users. In the control group, standard dosing will be continued. Rapid tests will be performed for FC every 4 weeks and for antiTNF trough levels every 12 weeks. The primary outcome is the cumulative incidence of out-of-range FC results at 48-week follow-up. Secondary endpoints include time to get out-of-range FC results, cumulative incidence of adverse effects, proportion of patients progressing to loss of response and identification of predictors of successful interval lengthening.

Ethics and dissemination The protocol has been approved by the Medical Ethics Review Committee of the University Medical Centre Groningen and is pending at the other participating centres. Results will be disseminated in peer-reviewed journals and presented at scientific meetings.

Trial registration number EudraCT number: 2020-001811-26; ClinicalTrials.gov Identifier: NCT04646187. Protocol version 4, date 17 September 2021.
Strengths and limitations of this study

This is the first prospective, interventional study to evaluate the feasibility of anti-tumour necrosis factor (TNF) dosing interval lengthening in a cohort of adolescents and young adults with inflammatory bowel disease.

- Potential harm of interval lengthening (lower antiTNF trough levels leading to pharmacokinetic loss of response) is minimised by 4-weekly monitoring of faecal calprotectin levels, which will detect an imminent flare at an early stage and thus allows proactive reversal to the original dosing interval.

- The partially randomised, patient preference design accommodates the patients (and their parents) with a strong preference for either interval lengthening or standard dosing.

- We acknowledge that the inclusion of nonrandomised patients creates a cohort study alongside a randomised controlled trial, which could compromise internal validity. Separate analyses of baseline characteristics and outcomes for both subsets of patients will reveal whether bias has occurred.

\section{INTRODUCTION}

Inflammatory bowel diseases (IBD), including Crohn's disease (CD) and ulcerative colitis (UC), are immune-mediated chronic relapsing disorders that often begin in adolescence or early adulthood. In both $\mathrm{CD}$ and $\mathrm{UC}$, the disease is characterised by bouts of inflammation (relapses or flares) and periods of remission. Active inflammation that goes untreated results in irreversible bowel damage for which intestinal surgery may be required. 
In the last decade, anti-tumour necrosis factor (TNF) agents (such as infliximab and adalimumab) have greatly improved the outcomes of patients with IBD, particularly when administered early in the course of the disease. ${ }^{1-3}$ Sustained remission, that is, long-lasting absence of disease activity, has now become a realistic treatment target. Real-world evidence studies have shown that $67 \%-91 \%$ of paediatric patients and up to $66 \%$ of adult patients is in sustained remission 2 years after the initiation of anti-TNF therapy. ${ }^{4-6}$

However, long-term exposure to anti-TNF agents is also associated with dose-dependent susceptibility to infections and dermatological adverse effects. Once sustained remission is achieved, many patients therefore ask whether it is feasible to stop or taper anti-TNF therapy. In fact, optimising current treatment strategies is considered one of the gap areas that must be addressed to get closer to precision medicine in IBD care. ${ }^{7}$

Both patients and healthcare professionals often wish to stop or reduce exposure to medication, provided that the benefits outweigh the risks. Key considerations are the risk of disease relapse, and whether retreatment with the same or other drugs is successful if de-escalation fails. Complete withdrawal of anti-TNF therapy may not be realistic, even when the patient with IBD was treated effectively early after the diagnosis. ${ }^{8}$ Dosing interval lengthening, on the other hand, may be a feasible alternative for patients in sustained remission who wish to reduce exposure to anti-TNF agents. ${ }^{9}$ Preliminary, uncontrolled studies suggest that interval lengthening is feasible in a relevant proportion of patients with IBD, as long as faecal calprotectin (FC) measurements are performed periodically to guide therapeutic decisions and antidrug antibodies are not present. ${ }^{10}{ }^{11}$ Studies in patients with rheumatoid arthritis, an autoimmune disorder that has many parallels with IBD, also demonstrated the feasibility of anti-TNF de-escalation. ${ }^{12}$

\section{Preliminary studies on dosing interval lengthening}

In a large cohort of Belgian adult patients with $\mathrm{CD}(\mathrm{n}=898)$ on adalimumab maintenance therapy, Van Steenbergen $e t$ $a l$ selected 40 patients who had de-escalated from a 2-week to a 3-week adalimumab dosing interval. Compared with controls with an unchanged dosing interval, trough levels in these 40 patients had dropped significantly within 4 months, but this did not lead to clinical or biochemical changes. During a median follow-up of 24 months, $65 \%$ of the patients maintained clinical remission. Clinical relapse occurred significantly more frequently in patients with a lengthened interval compared with controls (30\% vs $3 \%$, respectively) and required reversal to a 2-week dosing interval. ${ }^{13}$ In a French observational study that followed patients with IBD after adalimumab interval lengthening to $40 \mathrm{mg}$ every 3 weeks, 17 of 56 patients (30\%) had reverted to the standard 2-week dosing interval because of insufficient clinical, biochemical and/or morphological disease control. This was successful in 16 of these 17 patients (94\%). Confirmation of transmural healing (by
MRI) or endoscopic remission in the year before interval lengthening decreased the risk of symptomatic flare after de-escalation with a factor five. ${ }^{14}$

A Belgian randomised controlled trial (RCT) evaluated the use of infliximab trough-level measurements to decide on the interval between infusions and demonstrated that a 12-week interval is feasible in a proportion of patients. ${ }^{15}$

\section{Relevance for practice}

Usually, de-escalation studies are not a research priority for pharmaceutical companies, and consequently industry-initiated RCTs are unlikely to take place.

If the effect of interval lengthening, as proposed in this study protocol, is non-inferior to standard dosing, we feel that it should be part of optimal IBD care.

Second, this study may provide additional support for the disease modification hypothesis, that states that chronicity of inflammation can be reduced with early aggressive therapy (ie, anti-TNF agents). ${ }^{16-18}$ Studies in young patients with juvenile idiopathic arthritis, who typically have a short disease history, have shown that anti-TNF therapy early in their disease course can create a window of opportunity to successfully de-escalate. ${ }^{19}$ It is therefore essential to include adolescents and young adults in de-escalation studies.

\section{Research in context: FC monitoring}

Achieving endoscopic (or mucosal) healing is regarded as the ideal therapeutic target in IBD, because its attainment is associated with favourable long-term outcomes. ${ }^{20}$ However, frequent endoscopic inspection to evaluate resolution of inflammation is impractical. Persistent low FC levels correspond well with endoscopic healing, as is shown in several observational paediatric and adult studies, and can therefore serve as a proxy for mucosal healing. ${ }^{21-25}$

In previously asymptomatic patients, increasing concentrations of FC that cross the upper limit of the target range predict clinical relapse in the following 2-3 months. ${ }^{26}$ Frequent monitoring of FC levels therefore allows early detection of a disease flare. The discussion about the best FC cut-off point for mucosal healing is ongoing. In this study, we will use $250 \mu \mathrm{g} / \mathrm{g}$ for patients with $\mathrm{CD}$ and 150 $\mu \mathrm{g} / \mathrm{g}$ for patients with UC, based on its correspondence with endoscopic remission. ${ }^{27-34}$

\section{Study objectives}

The aim of this study is to evaluate the effectiveness of interval lengthening versus standard dosing after achieving sustained disease remission in patients with IBD, during 1 year of follow-up.

Secondary objectives include the evaluation of (1) the success rate of reversal to standard dosing after a first outof-range FC result and (2) the cumulative incidence of anti-TNF associated adverse effects after interval lengthening compared with standard dosing.

\section{METHODS AND ANALYSIS}

\section{Study design}

We designed a prospective, partially randomised patient preference trial that will run in multiple European 


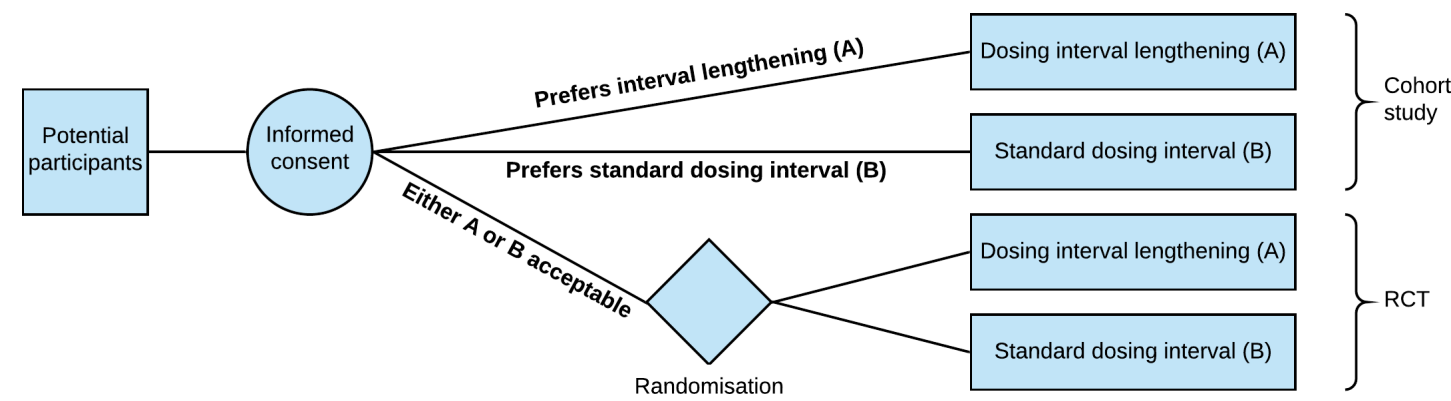

Figure 1 Allocation process. RCT, randomised controlled trial.

centres. Patients will be offered randomisation, but those with strong preferences can choose a strategy instead, and will be followed up identically. Hence, in this trial that compares (A) the lengthened dosing interval with (B) standard dosing interval, we will have four groups: randomised to $\mathrm{A}$; randomised to $\mathrm{B}$; prefer $\mathrm{A}$; prefer $\mathrm{B}$ (figure 1). Inclusion of non-randomised patients creates a cohort study alongside an RCT.

The first patient was included on 11 March 2021. Follow-up of the last patient is scheduled to end in the first quarter of 2023.

\section{Inclusion criteria}

Eligible patients are 12-25 years old, diagnosed with luminal CD or UC, treated with either 8-weekly infliximab or 2-weekly adalimumab as first ever anti-TNF agent (or as second anti-TNF agent for reasons other than primary nonresponse or secondary loss of response) and no previous attempts to lengthen the dosing interval. At study entry, patients should be in sustained remission, defined as the absence of symptoms of active IBD, combined with three consecutive FC results in the target range (i.e. $<250 \mu \mathrm{g} / \mathrm{g}$ for patients with $\mathrm{CD} ;<150 \mu \mathrm{g} / \mathrm{g}$ for patients with UC) in the previous 6 months, or combined with confirmed endoscopic remission in the last 2 months before study entry (ie, simple endoscopic score for Crohn's disease $<3$ points; UC endoscopic index of severity $\leq 1$ point or Mayo endoscopic subscore $\leq 1$ point).

\section{Exclusion criteria}

Potential participants will be excluded from the study if any of the following conditions occur: perianal fistula, presence of ileostomy or ileoanal pouch (as FC cut-off is not validated for small bowel faeces), any inflammatory comorbidity (such as rheumatoid arthritis), cotreatment with corticosteroids (prednisone or budesonide) or pregnancy.

\section{Intervention group}

In patients allocated to the intervention group, the interval between consecutive anti-TNF administrations will be lengthened. The individual dose itself and the choice for either infliximab or adalimumab will remain unaltered. In patients treated with adalimumab, the dosing interval will be lengthened from 2 to 3 weeks. In patients treated with infliximab, the dosing interval will be lengthened from 8 to 12 weeks.

\section{Control group}

Patients in the control group will continue on the standard dosing interval of 2 weeks for adalimumab and 8 weeks for infliximab. The dose and choice of anti-TNF agent will remain unaltered.

\section{Use of cointervention}

In addition to the anti-TNF agent, stable doses of concomitant maintenance medication will be continued in both groups and include immunomodulators (mercaptopurine, azathioprine, thioguanine or methotrexate) and/or aminosalicylates (sulfasalazine or mesalamine).

During the study period, the use of anti-TNF agents other than infliximab or adalimumab and the use of any investigational drug of chemical or biological nature other than the investigational medicinal products is prohibited, as is participation in other interventional studies.

\section{Primary outcome}

The primary outcome is the cumulative incidence of outof-range FC results at 48-week follow-up. Out-of-range FC results are defined as $\mathrm{FC}$ above the target range (i.e. $>250$ $\mu \mathrm{g} / \mathrm{g}$ for patients with $\mathrm{CD} ;>150 \mu \mathrm{g} / \mathrm{g}$ for patients with $\mathrm{UC}$ ) and at least $100 \mu \mathrm{g} / \mathrm{g}$ increase compared with the previous result, unless the previous result was already above the target range.

\section{Secondary outcomes}

Secondary endpoints include (1) time to get out-of-range FC results, defined as the time from study baseline until the first out-of-range FC result as defined above and (2) cumulative incidence of infections and dermatological adverse effects (eg, skin infections, psoriasis, eczema) at 48-week follow-up. ${ }^{35-37}$

Other secondary endpoints are (3) evolution of FC and anti-TNF trough levels in the first 16 weeks after reversal to standard dosing, (4) proportion of patients developing loss of response in the first 16 weeks after reversal to standard dosing, in which loss of response is defined as the appearance of symptoms of active IBD in combination with persistent out-of-range FC results, and (5) identification of predictors of successful de-escalation.

\section{Other outcomes}

To obtain insight into the attitude towards deprescribing anti-TNF agents, we will use the revised Patients' Attitudes Towards Deprescribing (rPATD) questionnaire. 
If an out-of-range FC result occurs, faeces of UMCGbased patients will be tested for colon pathogens (Salmonella enterica, Campylobacter jejuni, Shigella spp, Shiga toxin-producing Escherichia coli, Clostridium difficile and Cryptosporidium spp) to control for false-positive FC results.

\section{Sample size}

Based on analysis of real-life data in a historical cohort from the coordinating centre (University Medical Centre Groningen, UMCG) the annual baseline risk of out-ofrange FC results in young patients with IBD after reaching sustained remission on anti-TNF therapy is $20 \%$.

Interval lengthening is non-inferior to standard dosing if the cumulative incidence of out-of-range FC results (and its 95\% CI) is less than $+20 \%$ different from the control group. This non-inferiority margin corresponds to a $20 \%$ increase in out-of-range $\mathrm{FC}$ results and should not be confused with a $20 \%$ increase in symptomatic flares. We anticipate that $<10 \%$ of those who develop outof-range FC results will ultimately progress to secondary loss of response to anti-TNF therapy. We wish to detect this difference by a one-sided test at $2.5 \%$ level of significance with a probability of $80 \%$, a non-inferiority margin of $20 \%$, and a 1:1 allocation ratio. With the binary outcome (out-of-range result or not) 64 patients per group will be needed. To accommodate dropouts (5\%) and control for potential confounders caused by the inclusion of nonrandomised patients (10\%), we adjusted the sample size to a total of 74 patients per group.

\section{Allocation}

Patients will be recruited from both paediatric and adult IBD clinics from university hospitals in the Netherlands, Spain and Belgium.

As mentioned above, patients will be offered randomisation, but those with strong preferences can choose a strategy instead and will be followed-up identically. In the group of patients that are willing to be randomised, block randomisation with a variable block size (between 4 and 8) stratified for study centre will be performed in a 1:1 ratio. The allocation sequence is generated by the biostatistics unit of the UMCG, and is not available to any member of the research team. Allocation concealment will be ensured, as the REDCap study website will not release the randomization code until the teenager has been recruited into the trial ${ }^{38}{ }^{39}$ The nature of the intervention (lengthening of the dosing interval) does not allow blinding of the different strategies.

\section{Study procedures}

\section{Screening}

Two weeks prior to baseline, potential participants will be assessed for eligibility. Oral and written information about the study will be provided by the local IBD-team. Patients who do not meet inclusion criteria or who decline to participate, will be recorded anonymously, including patient characteristics and, if available, the reason of non-participation.

\section{Enrolment/baseline assessment}

After obtaining written informed consent, each patient will complete the rPATD questionnaire ${ }^{40-43}$ and additional questions about anti-TNF de-escalation and a baseline assessment will be performed. FC, anti-TNF trough levels and $\mathrm{C}$ reactive protein will be measured (figure 2).

\section{Post-allocation follow-up}

Patients will be followed until 48 weeks after study enrolment, or until 16 weeks after the first out-of-range FC. FC will be measured every 4 weeks with a validated point-of-care test ${ }^{44}$ (IBDoc, Bühlmann Laboratories AG, Schönenbuch, Switzerland) and a software application that turns a personal smartphone camera into a reader for quantitative measurements. At the same time, patients will self-monitor their symptoms of (impending) relapse, including abdominal pain, rectal bleeding, semiformed or liquid stool consistency, increased defecation frequency, nocturnal defecation, a decline in energy level and a decrease of appetite. ${ }^{46-49}$ Patients are instructed to contact their local IBD team if symptoms return between preset face-to-face encounters.

Adverse effects of anti-TNF will be assessed by selfreporting at weeks 12 and 36, and by the physician during face-to-face encounters at weeks 24 and 48. Anti-TNF trough levels will be measured every 12 weeks after interval lengthening and every 24 weeks in the cohort with standard dosing.

As soon as an out-of-range FC result is detected, treatment will be intensified according to protocol. In the intervention group, this primarily consists of reversal to standard anti-TNF dosing. After an out-of-range FC result, patients are followed for another 16 weeks, after which participation to the study is terminated. Follow-up after an out-of-range FC result consists of self-assessment of symptoms of relapse and FC testing every 4 weeks, and a face-to-face evaluation with anti-TNF trough level measurement every 8 weeks.

\section{Adherence and retention}

In this study, adherence refers to the degree to which patients act on reminders that it is time to perform a next FC measurement and complete a next online questionnaire. Low adherence can have a substantial effect on the interpretation of the study results. To help avoid these potential detrimental effects of non-adherence, we have implemented the following procedures: (1) reminding patients automatically (by email) to complete a questionnaire and to perform an FC measurement. This reminder is resent every 3 days for up to three times if a participant has not responded. (2) Mentioning the importance of keeping to agreements in the written patient information that patients receive upfront. (3) Emphasising the importance of keeping to agreements during the face-toface baseline evaluation. (4) If applicable, discussing the 


\begin{tabular}{|c|c|c|c|c|c|c|c|c|c|c|c|c|c|c|}
\hline \multirow[b]{3}{*}{ TIMEPOINT } & \multicolumn{14}{|c|}{ STUDY PERIOD } \\
\hline & \multirow{2}{*}{$\frac{\text { Enrolment }}{-t_{1}}$} & \multirow{2}{*}{$\frac{\text { Allocation }}{0}$} & \multicolumn{11}{|c|}{ Post-allocation (4-week intervals) } & \multirow{2}{*}{$\frac{\text { Close-out }}{t_{12}^{*}}$} \\
\hline & & & $t_{1}$ & $t_{2}$ & $t_{3}$ & $t_{4}$ & $t_{5}$ & $t_{6}$ & $t_{7}$ & $t_{8}$ & $t_{9}$ & $t_{10}$ & $t_{11}$ & \\
\hline \multicolumn{15}{|l|}{ ENROLMENT: } \\
\hline Eligibility screen & $x$ & $x$ & & & & & & & & & & & & \\
\hline Allocation & & $x$ & & & & & & & & & & & & \\
\hline \multicolumn{15}{|l|}{ INTERVENTIONS: } \\
\hline $\begin{array}{l}\text { Dosing interval } \\
\text { lengthening }\end{array}$ & & $\rightarrow$ & $\rightarrow$ & $\rightarrow$ & $\rightarrow$ & $\rightarrow$ & $\rightarrow$ & $\rightarrow$ & $\rightarrow$ & $\rightarrow$ & $\rightarrow$ & $\rightarrow$ & $\rightarrow$ & \\
\hline rPATD questionnaire & & $x$ & & & & & & & & & & & & \\
\hline Symptoms of relapse $(y / n)$ & & & $x$ & $x$ & $x$ & $x$ & $x$ & $x$ & $x$ & $x$ & $x$ & $x$ & $x$ & $x$ \\
\hline Adverse effects & & $x$ & & & $x$ & & & $x$ & & & $x$ & & & $x$ \\
\hline Faecal calprotectin & & $x$ & $x$ & $x$ & $x$ & $x$ & $x$ & $x$ & $x$ & $x$ & $x$ & $x$ & $x$ & $x$ \\
\hline Anti-TNF trough level & & $x$ & & & $x^{\dagger}$ & & & $x$ & & & $x^{+}$ & & & $x$ \\
\hline$D E$ or IS required $(y / n)$ & & & $x$ & $x$ & $x$ & $x$ & $x$ & $x$ & $x$ & $x$ & $x$ & $x$ & $x$ & $x$ \\
\hline $\begin{array}{l}\text { Patient refuses further } \\
\text { participation }(y / n)\end{array}$ & & & $x$ & $x$ & $x$ & $x$ & $x$ & $\mathrm{x}$ & $x$ & $x$ & $x$ & $x$ & $x$ & $X$ \\
\hline
\end{tabular}

Figure 2 Schedule of enrolment, interventions, and assessments. Black arrows=scheduled start of intervention; grey arrows=continuation, unless faecal calprotectin is out of range; DE, dose escalation; IS, interval shortening; rPATD, revised Patients' Attitudes Towards Deprescribing. * or 16 weeks after first out-of-range $\mathrm{FC} .{ }^{\dagger}$ only in the intervention group.

reason(s) for non-adherence with the patient, either by phone or during a face-to-face evaluation.

The study sites will make every reasonable effort to follow the participants for the entire study period. If a patient is lost to follow-up or withdraws from the study, data that have already been collected until that point will be used in the analyses. If available, a reason for withdrawal will be recorded.

\section{Confidentiality and data management}

Patients will receive a study ID number at enrolment. All data will be entered and stored linked to this study ID number. All study-related information will be securely stored electronically or at the study site. Patient information will be stored in electronic Case Report Forms in REDCap or in locked file cabinets if electronic storage is not possible.

Questionnaires will be completed digitally via a hyperlink sent by the REDCap study website. This will automatically be linked to the patient's study ID number.

Data will be stored during the study period and 25 years thereafter. If patients (and their parents in case of minors) give permission, residual serum will be stored for a maximum of 15 years at the local study site for future research.

\section{Data monitoring}

An independent Data Safety Monitoring Board (DSMB) has been established. The DSMB consists of three members: Thalia Hummel, MD PhD (chair, paediatric gastroenterologist at Medical Spectrum Twente, Enschede, The Netherlands), Anke Heida, MD PhD (epidemiologist at UMCG) and Douwe Postmus, PhD (statistician at UMCG). The DSMB charter and responsibilities of the DSMB are available on request.

The DSMB will receive results of an interim analysis in confidentiality when $50 \%$ of the participants have completed follow-up. Based on this interim analysis, the DSMB will advise as to whether the detected risk levels are acceptable.

The advice of the DSMB will only be sent to the sponsor. Should the sponsor decide not to fully implement the advice of the DSMB, the sponsor will send the advice to the reviewing Medical Research Ethics Committee (MREC), including a note to substantiate why (part of) the advice of the DSMB will not be followed.

\section{Adverse events}

In this study, adverse events are defined as any undesirable experience occurring to a patient during the study, whether or not considered related to the investigational 
product or the experimental intervention. All adverse events reported spontaneously by the subject or observed by the investigator or his staff after enrolment will be recorded. Adverse events that meet the criteria of serious adverse events will be reported to the accredited MREC according to the regulations of the concerned country.

\section{Patient and public involvement}

In 2015, the structure of the James Lind Alliance Priority Setting Partnership was used to identify and prioritise unanswered questions about treatments for IBD. This process culminated in a Top 10 of Research priorities. Priority number 1 is about the optimal treatment strategy, selecting the right patient group, the right stage of the disease and assessing the potential for withdrawal. ${ }^{5051}$

\section{Statistical analysis}

Data analysis will be coordinated by a statistician from the biostatics unit of the UMCG. The primary analyses will be conducted according to intention to treat. Secondary analyses will be conducted on a per-protocol base. A priori subgroup analyses will be performed to evaluate the effect of dose interval lengthening in two subtypes of IBD (CD and UC) and for both anti-TNF agents (infliximab and adalimumab). Baseline characteristics and outcomes of the randomised group will be compared with the combined randomised and preference group. Descriptive statistics will be used to compare baseline characteristics per allocated arm. Random missing data will be handled by using the multiple imputation Hot Deck method. The threshold for significance is set at $5 \%(\mathrm{p}<0.05)$.

Differences in cumulative incidence of out-of-range FC results between groups at 48 weeks will be analysed with a logistic regression model. Time-to-out-of-range FC results will be visualised with Kaplan-Meier curves. The HR with its $95 \%$ CI for out-of-range FC results will be provided with a Cox proportional hazards (multivariate) regression analysis. Potential confounding factors, including age at diagnosis and relapse-free interval before study enrolment will be included in the Cox proportional hazards model.

Statistical comparisons of the baseline characteristics and other secondary outcome measures will be analysed by means of independent samples t-tests, $\chi^{2}$ test, or MannWhitney tests, where appropriate. Predictors of successful de-escalation will be assessed by calculating ORs with the use of univariate logistic regression analysis. Candidate predictors with $\mathrm{p}<0.10$ in univariate analysis will be selected for use in the multivariate analysis. An interim safety analysis will be performed by an independent biostatistician when $50 \%$ of patients have completed the study.

\section{DISCUSSION}

With this study, we aim to determine whether the interval between consecutive anti-TNF administrations can be prolonged by $50 \%$ without compromising disease control in patients with IBD who have reached sustained clinical remission. The level of disease control will be tightly monitored via 4-weekly FC screening: out-of-range calprotectin levels will prompt reversal of the anti-TNF treatment interval to the standard dosing interval.

To date, convincing scientific evidence on the feasibility of anti-TNF interval lengthening is lacking. At the same time, patients often wish to stop or reduce exposure to this medication, in particular when they experience side effects associated with long-term exposure. Bridging this knowledge gap is an important step towards optimised IBD care. Provided that disease control is not jeopardised, interval lengthening of anti-TNF medication reduces the number of hospital visits for infliximab and the number of intravenous or subcutaneous administration of anti-TNF agents. In addition, interval lengthening can be expected to reduce anti-TNF associated side-effects as well as medical expenditure.

This study is designed as a partially randomised patientpreference trial. This design combines the methodology of an RCT and a patient preference clinical trial. ${ }^{52}$ Patients will be offered randomisation, but those with strong preferences can choose a strategy instead. Inclusion of non-randomised patients in our study will offer some reassurance that the results can be extrapolated to a wider group of patients. ${ }^{53}$ A potential limitation of the partially randomised patient preference design is that it may compromise internal validity. Separate analyses will therefore be performed on the randomised and the preference group.

\section{ETHICS AND DISSEMINATION}

This study will be conducted in accordance with the study protocol and the principles of the Declaration of Helsinki (version 2013). ${ }^{54}$ The study protocol has been approved at the primary site by the Medical Ethics Review Committee of the UMCG (METc 2020/340). At the secondary sites, seeking ethical approval is ongoing. In case of important protocol amendments, the Research Ethics Boards will be informed and the clinical trial registry will be updated. Written informed consent will be obtained from all patients and parents/legal guardians of minor patient prior to enrolment. Patients will be permitted to withdraw from the study at any time.

Any modifications to the protocol which may impact on the conduct of the study, potential benefit of the patient or may affect patient safety, including changes of study objectives, study design, patient population, sample sizes, study procedures, or significant administrative aspects will require a formal amendment to the protocol. Such amendment will be approved by the MREC prior to implementation and notified to the participating centres.

Results of the study will be disseminated in peerreviewed journals and presented at scientific meetings. 
Author affiliations

${ }^{1}$ Department of Paediatric Gastroenterology, Hepatology and Nutrition, University Medical Centre Groningen, Beatrix Childrens Hospital, Groningen, The Netherlands ${ }^{2}$ Department of Gastroenterology and Hepatology, University Medical Centre Groningen, Groningen, The Netherlands

${ }^{3}$ Department of Gastroenterology, University Hospital Ghent, Gent, Belgium ${ }^{4}$ Department of Gastroenterology, Central University Hospital of Liege, Liege, Belgium

${ }^{5}$ Department of Paediatric Gastroenterology, University Hospital Ghent, Gent, Belgium

${ }^{6}$ Department of Epidemiology, University Medical Centre Groningen, Groningen, The Netherlands

${ }^{7}$ Department of Gastroenterology, Bellvitge University Hospital, IDIBELL, L'Hospitalet de Llobregat, Spain

Twitter Henk Groen @Groen62H, Jordi Guardiola @JordiGuardiolac and Patrick van Rheenen @PFvRheenen

Contributors GD, TL, JG, EL, SvB and PvR initiated this study. MB and PvR drafted, reviewed and revised the manuscript. GD, TL, JG, EL, SvB and WSL reviewed and revised the manuscript. HG provided statistical expertise in clinical trial design. Al authors approved the final manuscript as submitted and agree to be accountable for all aspects of the work.

Funding This work was supported by a grant from the European Crohn's and Colitis Organisation (ECCO 2021). Materials for the calprotectin and anti-TNF trough level point-of-care tests are donated by BÜHLMANN Laboratories AG (Schönenbuch, Switzerland). Both funding sources had no role in the design of this study and will not have any role during its execution, analyses, interpretation of the data, or decision to submit results.

\section{Competing interests None declared.}

Patient and public involvement Patients and/or the public were not involved in the design, or conduct, or reporting, or dissemination plans of this research.

Patient consent for publication Not applicable.

Provenance and peer review Not commissioned; externally peer reviewed.

Open access This is an open access article distributed in accordance with the Creative Commons Attribution Non Commercial (CC BY-NC 4.0) license, which permits others to distribute, remix, adapt, build upon this work non-commercially, and license their derivative works on different terms, provided the original work is properly cited, appropriate credit is given, any changes made indicated, and the use is non-commercial. See: http://creativecommons.org/licenses/by-nc/4.0/.

\section{ORCID iDs}

Marleen Bouhuys http://orcid.org/0000-0001-6530-2198

Henk Groen http://orcid.org/0000-0002-6629-318X

Patrick van Rheenen http://orcid.org/0000-0003-3867-2665

\section{REFERENCES}

1 Schreiber S, Reinisch W, Colombel JF, et al. Subgroup analysis of the placebo-controlled CHARM trial: increased remission rates through 3 years for adalimumab-treated patients with early Crohn's disease. J Crohns Colitis 2013;7:213-21.

2 Torres J, Bonovas S, Doherty G, et al. ECCO guidelines on therapeutics in Crohn's disease: medical treatment. J Crohns Colitis 2020;14:4-22.

3 Ungaro RC, Aggarwal S, Topaloglu O, et al. Systematic review and meta-analysis: efficacy and safety of early biologic treatment in adult and paediatric patients with Crohn's disease. Aliment Pharmacol Ther 2020;51:831-42.

4 van Rheenen $\mathrm{H}$, van Rheenen PF. Long-Term efficacy of antitumor necrosis factor agents in pediatric luminal Crohn's disease: a systematic review of real-world evidence studies. Pediatr Gastroenterol Hepatol Nutr 2020;23:121.

5 Bressler B, Mantzaris G, Silverberg M, et al. P621 real-world effectiveness and safety of vedolizumab and anti-TNF in biologicnaive Crohn's disease patients: results from the EVOLVE study. $J$ Crohns Colitis 2019;13:S427-8.

6 Yarur A, Mantzaris G, Silverberg M, et al. P573 real-world effectiveness and safety of vedolizumab and anti-TNF in biologicnaive ulcerative colitis patients: results from the evolve study. $J$ Crohns Colitis 2019;13:S400-1.
7 Denson LA, Curran M, McGovern DPB, et al. Challenges in IBD research: precision medicine. Inflamm Bowel Dis 2019;25:S31-9.

8 Gisbert JP, Marín AC, Chaparro M. The risk of relapse after anti-TNF discontinuation in inflammatory bowel disease: systematic review and meta-analysis. Am J Gastroenterol 2016;111:632-47.

9 Doherty G, Katsanos KH, Burisch J, et al. European crohn's and colitis organisation topical review on treatment withdrawal ['exit strategies'] in inflammatory bowel disease. J Crohns Colitis 2018;12:17-31.

10 Papamichael K, Karatzas P, Mantzaris GJ. De-escalation of infliximab maintenance therapy from 8- to 10-week dosing interval based on faecal calprotectin in patients with Crohn's disease. J Crohns Colitis 2016;10:371-2

11 Buurman DJ, Maurer JM, Keizer RJ, et al. Population pharmacokinetics of infliximab in patients with inflammatory bowel disease: potential implications for dosing in clinical practice. Aliment Pharmacol Ther 2015;42:529-39.

12 Henaux S, Ruyssen-Witrand A, Cantagrel A, et al. Risk of losing remission, low disease activity or radiographic progression in case of bDMARD discontinuation or tapering in rheumatoid arthritis: systematic analysis of the literature and meta-analysis. Ann Rheum Dis 2018;77:515-22.

13 Van Steenbergen S, Bian S, Vermeire S, et al. Dose de-escalation to adalimumab $40 \mathrm{mg}$ every 3 weeks in patients with Crohn's disease - a nested case-control study. Aliment Pharmacol Ther 2017;45:923-32.

14 Pouillon L, Lamoureux A, Pineton de Chambrun G, et al. Dose de-escalation to adalimumab $40 \mathrm{mg}$ every three weeks in patients with inflammatory bowel disease-A multicenter, retrospective, observational study. Dig Liver Dis 2019;51:236-41.

15 Vande Casteele N, Ferrante M, Van Assche G, et al. Trough concentrations of infliximab guide dosing for patients with inflammatory bowel disease. Gastroenterology 2015;148:1320-9.

16 Solitano V, D'Amico F, Zacharopoulou E, et al. Early intervention in ulcerative colitis: ready for prime time? J Clin Med 2020;9. doi:10.3390/jcm9082646. [Epub ahead of print: 1408 2020].

17 Danese S, Fiorino G, Peyrin-Biroulet L. Early intervention in Crohn's disease: towards disease modification trials. Gut 2017;66:2179-87.

18 Yzet C, Ungaro R, Bossuyt P, et al. OP35 endoscopic and deep remission at 1 year prevents disease progression in early crohn's disease: long-term data from CALM. J Crohns Colitis 2019;13:S024-5.

19 Wallace CA, Giannini EH, Spalding SJ, et al. Trial of early aggressive therapy in polyarticular juvenile idiopathic arthritis. Arthritis Rheum 2012;64:2012-21.

20 Peyrin-Biroulet L, Sandborn W, Sands BE, et al. Selecting therapeutic targets in inflammatory bowel disease (STRIDE) determining therapeutic goals for treat-to-target. Am J Gastroenterol 2015:110:1324-38

21 Kolho K-L, Sipponen T. The long-term outcome of anti-tumor necrosis factor- $\alpha$ therapy related to fecal calprotectin values during induction therapy in pediatric inflammatory bowel disease. Scand $J$ Gastroenterol 2014;49:434-41.

22 Zubin G, Peter L. Predicting endoscopic crohn's disease activity before and after induction therapy in children: a comprehensive assessment of PCDAI, CRP, and fecal calprotectin. Inflamm Bowel Dis 2015;21:1386-91.

23 Ziv-Baran T, Hussey S, Sladek M, et al. Response to treatment is more important than disease severity at diagnosis for prediction of early relapse in new-onset paediatric crohn's disease. Aliment Pharmacol Ther 2018;48:1242-50.

24 Louis E, Mary J-Y, Vernier-Massouille G, et al. Maintenance of remission among patients with crohn's disease on antimetabolite therapy after infliximab therapy is stopped. Gastroenterology 2012;142:e5:63-70

25 Guardiola J, Lobatón T, Cerrillo E, et al. Recommendations of the spanish working group on crohn's disease and ulcerative colitis (GETECCU) on the utility of the determination of faecal calprotectin in inflammatory bowel disease. Gastroenterol Hepatol 2018;41:514-29.

26 Heida A, Park KT, van Rheenen PF. Clinical utility of fecal calprotectin monitoring in asymptomatic patients with inflammatory bowel disease: a systematic review and practical guide. Inflamm Bowel Dis 2017;23:894-902.

27 Lobatón T, López-García A, Rodríguez-Moranta F, et al. A new rapid test for fecal calprotectin predicts endoscopic remission and postoperative recurrence in crohn's disease. J Crohns Colitis 2013;7:e641-51.

28 Plevris N, Fulforth J, Lyons M, et al. Normalization of fecal calprotectin within 12 months of diagnosis is associated with reduced risk of disease progression in patients with crohn's disease. Clin Gastroenterol Hepatol 2021;19:1835-1844.e6. 
29 Lobatón T, Rodríguez-Moranta F, Lopez A, et al. A new rapid quantitative test for fecal calprotectin predicts endoscopic activity in ulcerative colitis. Inflamm Bowel Dis 2013;19:1034-42.

30 Guardiola J, Lobatón T, Rodríguez-Alonso L, et al. Fecal level of calprotectin identifies histologic inflammation in patients with ulcerative colitis in clinical and endoscopic remission. Clin Gastroenterol Hepatol 2014;12:1865-70.

31 Colombel J-F, Panaccione R, Bossuyt P, et al. Effect of tight control management on crohn's disease (calm): a multicentre, randomised, controlled phase 3 trial. Lancet 2017;390:2779-89.

32 Kristensen V, Røseth A, Ahmad T. Fecal calprotectin: a reliable predictor of mucosal healing after treatment for active ulcerative colitis. Gastroenterol Res Pract 2017;2017:2098293-5.

33 Jauregui-Amezaga A, López-Cerón M, Aceituno M, et al. Accuracy of advanced endoscopy and fecal calprotectin for prediction of relapse in ulcerative colitis: a prospective study. Inflamm Bowel Dis 2014;20:1187-93.

34 Zhulina Y, Cao Y, Amcoff K, et al. The prognostic significance of faecal calprotectin in patients with inactive inflammatory bowel disease. Aliment Pharmacol Ther 2016;44:495-504.

35 European Medicines Agency. Remicade $100 \mathrm{mg}$ powder for concentrate for solution for infusion - summary of product characteristics (SPC) - (eMC), 2009. Available: https://www.ema europa.eu/en/documents/product-information/remicade-eparproduct-information_en.pdf [Accessed 31 Mar 2020].

36 European Medicines Agency. Humira $20 \mathrm{mg}$ solution for injection in pre-filled syringe - summary of product characteristics (SPC) - (eMC) 2008. Available: https://www.ema.europa.eu/en/documents/productinformation/humira-epar-product-information_en.pdf [Accessed 31 Mar 2020].

37 Shivaji UN, Sharratt CL, Thomas T, et al. Review article: managing the adverse events caused by anti-TNF therapy in inflammatory bowel disease. Aliment Pharmacol Ther 2019;49:664-80.

38 Harris PA, Taylor R, Minor BL, et al. The REDCap consortium: building an international community of software platform partners. $J$ Biomed Inform 2019;95:103208.

39 Harris PA, Taylor R, Thielke R, et al. Research electronic data capture (REDCap)--a metadata-driven methodology and workflow process for providing translational research informatics support. J Biomed Inform 2009;42:377-81.

40 Reeve E, Low L-F, Shakib S, et al. Development and validation of the revised patients' attitudes towards deprescribing (rPATD) questionnaire: versions for older adults and caregivers. Drugs Aging 2016;33:913-28.
41 Edelman M, Jellema P, Hak E, et al. Patients' attitudes towards deprescribing alpha-blockers and their willingness to participate in a discontinuation trial. Drugs Aging 2019;36:1133-9.

42 Roux B, Sirois C, Niquille A, et al. Cross-cultural adaptation and psychometric validation of the revised patients' attitudes towards deprescribing (rPATD) questionnaire in French. Res Social Adm Pharm 2021:17:1453-62.

43 de Juan Roldán J, Gavilán Moral E, García Ruiz A. Estudio de validación al castellano del cuestionario revised patients' attitudes towards deprescribing (rPATD) para evaluar las actitudes de los pacientes hacia la deprescripción. Barcelona: XXXVIII Congreso semFYC, 2018.

44 Heida A, Knol M, Kobold AM, et al. Agreement between home-based measurement of stool calprotectin and ELISA results for monitoring inflammatory bowel disease activity. Clin Gastroenterol Hepatol 2017;15:1742-9.

45 Haisma S-M, Galaurchi A, Almahwzi S, et al. Head-to-head comparison of three stool calprotectin tests for home use. PLoS One 2019;14:e0214751.

46 Turner D, Otley AR, Mack D, et al. Development, validation, and evaluation of a pediatric ulcerative colitis activity index: a prospective multicenter study. Gastroenterology 2007;133:423-32.

47 Walmsley RS, Ayres RC, Pounder RE, et al. A simple clinical colitis activity index. Gut 1998;43:29-32.

48 Hyams JS, Ferry GD, Mandel FS, et al. Development and validation of a pediatric Crohn's disease activity index. J Pediatr Gastroenterol Nutr 1991;12:439-47.

49 Lewis JD, Chuai S, Nessel L, et al. Use of the noninvasive components of the Mayo score to assess clinical response in ulcerative colitis. Inflamm Bowel Dis 2008;14:1660-6.

50 Hart AL, Lomer M, Verjee A, et al. What are the top 10 research questions in the treatment of inflammatory bowel disease? A priority setting partnership with the James Lind alliance. J Crohns Colitis 2017;11:204-11.

51 Geldof J, LeBlanc J-F, Lucaciu L, et al. Are we addressing the top 10 research priorities in IBD? Frontline Gastroenterol 2020;2:flgastro2020-101579.

52 Schultz KF, GD A. Boosting recruitment to randomised controlled trials. In: Essential concepts in clinical research. 2nd edn. Edinburgh: Elsevier limited, 2019: 112-3.

53 Rush AJ. STAR*D: what have we learned? Am J Psychiatry 2007;164:201-4.

54 World Medical Association Declaration of Helsinki. Ethica principles for medical research involving human subjects. Jama 2013;310:2191-4. 